\title{
Commentary on: drug-drug interactions and clinical considerations with co-administration of antiretrovirals and psychotropic drugs
}

\author{
Daniel Hernandez-Huerta (1)
}

Psychiatry Department, Hospital Universitario Ramón y Cajal, Madrid, Spain.

Received 26 November 2018; Accepted 1 December 2018; First published 27 March 2019

Key words: HIV, psychotropic drugs, antipsychotics, paliperidone, antiretroviral therapy, drug interactions.

Drs. Goodlet, Zmarlicka, and Peckham provide a thorough review of the psychopharmacologic drugs commonly used by people living with HIV/AIDS (PLWHA). In their comprehensive review, the authors highlight the currently available information about drug-drug interactions (DDIs) between antiretroviral therapy (ART) and psychotropic drugs used in the treatment of psychiatric disorders, the possible overlapping toxicities of both treatments, and provide recommendations for their management. ${ }^{1}$

Regarding antipsychotic drugs, in the article, it is emphasized that there is a paucity of available data for clinical information about DDIs between ART and antipsychotics. Generally, many of the antipsychotics are metabolized via the CYP P450 system, in particular CYP 3A4 and CYP 2D6, and the potential for DDIs exists with concomitant administration of medications affecting these enzymes. However, in this review, the drug paliperidone is not mentioned. This treatment is an antipsychotic that could have many advantages in PLWHA.

Paliperidone, or 9-hydroxy-risperidone, is the major active metabolite of risperidone. It is an atypical antipsychotic which has significantly evidenced to be effective in improving psychotic symptoms in both oral and depot long-acting intramuscular formulations. ${ }^{2,3}$ Although there are no studies about the use of paliperidone among PLWHA, it is remarkable that recent consensus document on psychiatric aspect in adults with HIV infection recommends, specifically, the use of paliperidone for psychosis. This document specifies that

Address correspondence to: Daniel Hernandez-Huerta, Psychiatry Department, Hospital Universitario Ramón y Cajal, Ctra. Colmenar Viejo, km 9.100, CP: 28034, Madrid, Spain. (Email: daniel.hernandez@) salud.madrid.org) paliperidone is the antipsychotic of choice due to its lower risk of extrapyramidal symptoms, metabolic side effects, and, mostly, pharmacokinetic interactions than other typical and atypical antipsychotics. ${ }^{4}$

The metabolism of paliperidone in the liver is very limited; approximately $60 \%$ of the molecule is eliminated, unaltered, in the urine. The rest is eliminated in the form of urinary metabolites through dealkylation (4.6\%), hydroxylation $(3.8 \%)$, dehydrogenation $(2.7 \%)$, and breakdown of the benzisoxazole nucleus $(4.1 \%)$, and a small amount in feces. Due to its negligible hepatic biotransformation, paliperidone is unlikely to be involved in clinically significant metabolic interactions. Even though in vitro studies have demonstrated that isoenzymes CYP2D6 and CYP3A4 can intervene in paliperidone metabolism, there are no in vivo data available to confirm whether or not said isoenzymes play a significant metabolic role. For example, in a study of five healthy volunteers, after a single 1-mg dose of immediate-release paliperidone was taken orally, no metabolite was detected in the plasma. There are additional properties in terms of its lower protein binding and decreased inhibition of P-glycoprotein that lead to a decreased potential for DDIs. ${ }^{5,6}$

Based on this data, it seems that paliperidone treatment, both the oral formulation and the depot longacting intramuscular formulation, could be an effective, tolerable, and safety treatment in PLWHA taking ART. However, as with the other antipsychotics, caution and close monitoring of side effects are recommended when prescribing paliperidone treatment to HIV-infected patients due to the paucity of studies. Randomized controlled trials are needed to establish both efficacy and safety of antipsychotic drugs in the HIV population in order to improve and optimize psychiatric care. 


\section{Disclosures}

Dr. Hernandez-Huerta reports travel, accommodations, and meeting expenses from Jannsen Pharmaceutical, Otsuka Pharmaceutical, and Lundbeck Pharmaceutical, outside the submitted work.

\section{REFERENCES:}

1. Goodlet KJ, Zmarlicka MT, Peckham AM. Drug-drug interactions and clinical considerations with co-administration of antiretrovirals and psychotropic drugs. CNS Spectr. October 2018; 1-26. doi: 10. 1017/S109285291800113X.

2. Kane J, Canas F, Kramer M, et al. Treatment of schizophrenia with paliperidone extended-release tablets: A 6-week placebo-controlled trial. Schizophr Res. 2007; 90(1-3): 147-161. doi: 10.1016/j.schres. 2006.09.012.
3. Gopal S, Hough DW, Xu H, et al. Efficacy and safety of paliperidone palmitate in adult patients with acutely symptomatic schizophrenia: A randomized, double-blind, placebo-controlled, dose-response study. Int Clin Psychopharmacol. 2010; 25(5): 247-256. doi: 10. 1097/YIC.0b013e32833948fa.

4. Polo R, Blanch J, Locutura J, Arazo P. Consensus document on psychiatric and psychological aspect in adults and children with HIV infection. Enferm Infecc Microbiol Clin. 2016; 34(1): 53.e1-53.e14. doi: 10.1016/j.eimc.2015.07.018.

5. Mora F, Molina JD, Zubillaga E, López-Muñoz F, Álamo C. CYP450 and its implications in the clinical use of antipsychotic drugs. Clin Exp Pharmacol. 2015; 5(176). doi: 10.4172/2161-1459. 1000176.

6. Vermeir M, Naessens I, Remmerie B, et al. Absorption, metabolism, and excretion of paliperidone, a new monoaminergic antagonist, in humans. Drug Metab Dispos. 2008; 36(4): 769-779. doi: 10.1124/ dmd.107.018275. 